\title{
The Evaluation of Meropenem Dosing Regimens Against ESBL-Producing Escherichia coli in ICU Patients Using Monte Carlo Simulation
}

\author{
Ei Ei Win (iD)', Khaing Win Htun², Pramote Tragulpiankit', Suwida Tangtrakultham', Preecha Montakantikul' \\ 'Division of Clinical Pharmacy, Department of Pharmacy, Faculty of Pharmacy, Mahidol University, Bangkok, 10400, Thailand; ${ }^{2}$ Right Laboratory and \\ Health Screen, Naypyitaw, Myanmar \\ Correspondence: Preecha Montakantikul, Division of Clinical Pharmacy, Department of Pharmacy, Faculty of Pharmacy, Mahidol University, Bangkok, \\ 10400, Thailand, Tel +66-26448694, Email preecha.mon@mahidol.ac.th
}

\begin{abstract}
Purpose: To evaluate the optimal dosing regimens of meropenem against extended-spectrum beta-lactamase-producing Escherichia coli (ESBL E. coli) in critically ill patients with varying degrees of renal function using Monte Carlo simulation (MCS).

Methods: The MCS was performed using the minimum inhibitory concentration (MIC) data from Right Laboratory and Health Screen in Naypyitaw, Myanmar, as well as reported meropenem pharmacokinetic parameters in the target population and the pharmacokinetic-pharmacodynamic index. For each dosing regimen, 10,000 virtual patients were generated to assess the probability of target attainment (PTA) and the cumulative fraction of response (CFR). The most effective dosage regimens were determined using PTA and a CFR of $90 \%$.

Results: ESBL E. coli made up 93 of the 396 clinical E. coli isolates, and they are all multidrug-resistant, with resistance to at least five antibiotic classes. The $\mathrm{MIC}_{50}$ and $\mathrm{MIC}_{90}$ were determined to be $0.25 \mu \mathrm{g} / \mathrm{mL}$. The PTA was affected by five factors: creatinine clearance (CLcr), vasopressor usage, MIC, infusion time, and dosage fractionation. In patients who did not receive vasopressors, the current regimens (US-FDA and EMA) were ineffective in all renal function for MIC $>0.25 \mu \mathrm{g} / \mathrm{mL}$. In the subset group of CLcr $>80 \mathrm{~mL} / \mathrm{min}$ for MIC $2 \mu \mathrm{g} / \mathrm{mL}$, the maximum total daily dose of $6 \mathrm{~g} /$ day $(2 \mathrm{~g} \mathrm{q} 8 \mathrm{hr} ; 3 \mathrm{hr}$ infusion) was still ineffective, but $4 \mathrm{~g} /$ day $(1 \mathrm{~g}$ q $6 \mathrm{hr} ; 3 \mathrm{hr}$ infusion) achieved $98.96 \%$ PTA. Almost majority of the simulated regimens produced $>90 \%$ PTA in vasopressor-dependent patients with all levels of renal function, resulting in a decreased total daily dose requirement.

Conclusion: For high MIC ( $>1 \mu \mathrm{g} / \mathrm{mL}$ ) patients who do not use vasopressors and have a CLcr $>80 \mathrm{~mL} / \mathrm{min}$, a combination of dosage fractionation and the extended infusion was considered as an effective technique to maximize target attainment. Neither prolonged infusion nor dosage fractionation should be explored in patients using vasopressors.
\end{abstract}

Keywords: carbapenem, gram-negative bacteria, PKPD, dosing simulation, critically ill patients

\section{Introduction}

Extended-spectrum $\beta$-lactamase-producing Escherichia coli (ESBL E. coli) is one of the most serious cases of beta-lactam antimicrobial resistance in the globe. Beta-lactam antimicrobials are routinely utilized by healthcare professionals in the treatment of numerous bacterial nosocomial and community illnesses. ${ }^{1}$ As a result of the limited treatment options, improper initial therapy, and delayed treatment, ESBL-producing E. coli causes the increased length of hospitalization, higher morbidity, and a high healthcare burden..$^{2-4}$ All of this put ESBL E. coli, a drug resistant Enterobacterial, on the World Health Organization (WHO)'s top 3 priority list for new treatments. ${ }^{4}$ Southeast Asia is included in the highest risk of antimicrobial resistance in the world due to the high population density, the high burden of diseases and the widespread use of antibiotics. ${ }^{5}$ In Myanmar, the prevalence of ESBL E. coli was found from $38 \%$ in 2014 to $49.1 \%$ in $2016 .{ }^{6,7}$

Meropenem is routinely used to treat serious enterobacterial infections that produce ESBLs. ${ }^{1}$ Meropenem dosing regimens recommended by the United States Food and Drug Administration (US-FDA), on the other hand, were less effective against bacteria categorized as susceptible by the Clinical and Laboratory Standards Institute (CLSI), especially 
in the intensive care unit (ICU). ${ }^{8}$ Pathophysiological alterations in ICU patients who are in a life-threatening situation influence the pharmacokinetic properties of meropenem, leading to sub-therapeutic therapy, treatment failure, or the growth of antibiotic resistance. ${ }^{9}$ Unless we can prevent and regulate the development of very resistant germs, patients afflicted with this pathogen may soon have limited therapeutic options. The most straightforward way to avoid this problem is to use existing antibiotics appropriately, taking into account the current minimum inhibitory concentration (MIC) and the patient's likely condition.

Monte Carlo simulation (MCS) is a statistical method for predicting and evaluating antimicrobial drug effectiveness and optimizing their administration scheme using antimicrobial pharmacokinetic parameters, covariate factors, the MIC of the microorganism, and the pharmacokinetic/pharmacodynamic (PKPD) index. ${ }^{9,10}$ MCS is a beneficial tool for clinicians and researchers to develop optimal empirical therapeutic regimens. ${ }^{11}$ Because individual therapeutic drug monitoring for meropenem is not yet commonly used, a pharmacokinetic and pharmacodynamic study using MCS is better suited to determining the best dose regimens for specific MICs and demographics. As a result, the purpose of the study is to determine the optimal meropenem dosing regimens for ESBL E. coli using the concept of the PK/PD of meropenem, the MCS process, and the local MIC of ESBL E. coli.

\section{Materials and Methods \\ Microbiology}

Microbiological data of E. coli (clinical isolate data and MICs) from 2018 to 2019 were retrospectively extracted from the electronic database of Right Laboratory and Health Screen, a private microbiology laboratory, Naypyidaw, Myanmar. The clinical specimens were part of the routine laboratory procedure, and the study accessed the microbiological data from the database with the permission of the dataset owner of the laboratory. The institutional review board of Defense Services Medical Research Center in Naypyitaw, Myanmar (Document No: IRB/2020/A-03) and Mahidol University's Faculty of Dentistry and Faculty of Pharmacy (Document No: 78.0319/517) both approved the use of laboratory microbiology data for the study. The antimicrobial susceptibility was tested using the Vitek 2-Biomerieux system, and MICs were assessed using the M100 CLSI guidelines of $2020 .^{12}$ If $E$. coli isolates were resistant to third-generation cephalosporins, they were then considered as ESBL producers. From the resulting $\mathrm{MIC}$ distribution, $\mathrm{MIC}_{50}$ and $\mathrm{MIC}_{90}$ of meropenem against ESBL E. coli were calculated.

\section{Monte Carlo Simulation}

The pharmacokinetic parameters of a previous two-compartment population-based pharmacokinetic experiment conducted in severely infected patients were used. ${ }^{13}$ The two most important factors impacting meropenem clearance (CL) were creatinine clearance (CLcr) and vasopressor (Vaso) administration, and we used these covariates to determine the optimum meropenem dosing regimens. We used the two-compartment infusion model to construct the plasma concentration-time profile of meropenem for each dosage regimen because the population model was fit to the twocompartment model.

The target PK/PD index of meropenem was obtained from Li et al study, which indicated the free time above the minimum inhibitory concentration ( $f \mathrm{~T}>\mathrm{MIC}$ ) of $54 \% .{ }^{14}$ The bactericidal pharmacodynamic breakpoint in EUCAST was defined as $f \mathrm{~T}>\mathrm{MIC} 54 \%$. Based on evidence resulted from in vitro, in vivo, and clinical pharmacodynamic research, this breakpoint was determined as the maximal bactericidal activity. Therefore, this breakpoint $(54 \% f \mathrm{~T}>\mathrm{MIC})$ was used in this study as the pharmacodynamic target to calculate PTA and CFR.

Based on the PK parameters and associated covariates, 10,000 virtual patients were generated in each dosage regimen using Oracle Crystal Ball ${ }^{\circledR}$ software (Oracle Crystal Ball ${ }^{\circledR}$ version 11.1.2.4.850) to build the drug plasma concentrationtime profile over 24 hours. All parameters were assumed to be distributed lognormally before simulation in 10,000 trials. After that, the PTA for each dosing regimen was calculated at every MIC range to determine what percentage of subjects achieved 54\% $f \mathrm{~T}>\mathrm{MIC}$ target. The CFR was then calculated based on the actual MIC distribution of meropenem against ESBL E. coli from the Right Laboratory and Health Screen. The dose regimens that achieved $90 \%$ PTA and $90 \%$ CFR were considered the optimum and recommended for documented and empirical treatment of ESBL E. coli. 
The meropenem dose regimens were from reference regimens developed by the US-FDA, EMA, Ehmann et al, as well as our determination. ${ }^{15-17}$ Only the dose regimens that cover the CLSI breakpoint from Ehmann et al were selected. Table 1 displays dose schedules range from a conventional dosing infusion $(30 \mathrm{~min})$ to a prolonged infusion ( $3 \mathrm{hr})$. These dose regimens were simulated for the patients with and without vasopressors at varying levels of renal function, ranging from CLcr $1-10 \mathrm{~mL} / \mathrm{min}$ to CLcr $81-100 \mathrm{~mL} / \mathrm{min}$.

\section{Results \\ Microbiology}

A total of 396 clinical E. coli isolates were extracted from 2018 to 2019. Of these, 93 (23.48\%) isolates were ESBLproducing and used for MIC distribution to determine the $\mathrm{MIC}_{50 / 90}$ of meropenem against ESBL E. coli. Of these 93 ESBL E. coli isolates, $52.68 \%(\mathrm{n}=49)$ were from urine and $47.32 \%$ were from non-urine sources consisting of sputum 6.45\% ( $\mathrm{n}=6)$; pus 19.35\% $(\mathrm{n}=18)$; blood 4.3\% $(\mathrm{n}=4)$; stool $2.1 \%(\mathrm{n}=2)$ and wound swab $15.05 \%(\mathrm{n}=14)$. The AST result of 93 ESBL E. coli isolates is presented in Table 2 and showed that all isolates were multidrugresistant and resistant to at least five antibiotic categories. However, all ESBL isolates were $100 \%$ sensitive to meropenem. High sensitivity values were also observed for other carbapenems (imipenem and ertapenem) at $100 \%$, amikacin at $96.8 \%$, and piperacillin/tazobactam at $75.3 \%$. The $\mathrm{MIC}_{50}$ and $\mathrm{MIC}_{90}$ of meropenem against ESBL E. coli were $0.25 \mu \mathrm{g} / \mathrm{mL}$.

\section{Monte Carlo Simulation \\ Probability of Target Attainment}

The PTA results of all simulated meropenem dosing regimens for vasopressor and non-vasopressor receiving patients with varying degrees of renal function are shown in Figures 1A-E and Figure 2A-E. Analysis of PTA suggested that the risk of target failure increases with increasing CLcr in patients not receiving vasopressors. At CLcr $>80 \mathrm{~mL} / \mathrm{min}$, almost all simulated 30-minute infusion regimens failed to reach the target at MIC of $>0.25 \mu \mathrm{g} / \mathrm{mL}$. It was found that the prolonged infusion of same total daily dose resulted in a higher PTA than the 30-minute infusion, and the PTA differences between these two infusion methods were evident when CLcr was $>50 \mathrm{~mL} / \mathrm{min}$. However, in the group with CLcr of $>80 \mathrm{~mL} / \mathrm{min}$, even the prolonged infusion of the maximum total daily dose of $6 \mathrm{~g} /$ day (the regimen of Ehmann et al; $2 \mathrm{~g} \mathrm{q} 8 \mathrm{hr}$ ) achieved only $81.64 \%$ PTA at MIC of $2 \mu \mathrm{g} / \mathrm{mL}$, whereas the fractionated regimen with the lower total daily dose of $4 \mathrm{~g}$ /day ( $1 \mathrm{~g} \mathrm{q} 6 \mathrm{~h}$ ) could achieve $>90 \%$ of the PTA. In contrast, most dosing regimens achieved higher PTA and maintained $>90 \%$ at all renal functions in patients receiving vasopressors. Even CLcr of $>80 \mathrm{~mL} / \mathrm{min}$, at least $2 \mathrm{~g} /$ day $(0.5 \mathrm{~g} \mathrm{q} 6 \mathrm{~h})$ and $1.5 \mathrm{~g} /$ day $(0.5 \mathrm{~g} \mathrm{q} 8 \mathrm{~h})$ were sufficient to achieve the target PTA at MIC of $2 \mu \mathrm{g} / \mathrm{mL}$ and $1 \mu \mathrm{g} /$ $\mathrm{mL}$, respectively. PTA differences in the prolonged and short infusion were not evident in this group of patients even at CLcr of $>80 \mathrm{~mL} / \mathrm{min}$. On the other hand, at the low MIC of $0.25 \mu \mathrm{g} / \mathrm{mL}$, a high PTA ( $>90 \%$ ) was achieved with the lower dosing requirement in both patient groups regardless of renal function.

\section{CFR}

The CFR results of meropenem against ESBL E. coli are summarized in Table 3. For the bactericidal goal of 54\% $f \mathrm{~T}>\mathrm{MIC}$ in patients not receiving vasopressors, all dosing regimens with the 30-minute infusion except $4 \mathrm{~g} / \mathrm{day}$ (1g q 6hr) were unable to reach $>90 \%$ CFR when CLcr is $>80 \mathrm{~mL} / \mathrm{min}$. In patients with CLcr of $<10 \mathrm{~mL} / \mathrm{min}$, at least $0.25 \mathrm{~g} /$ day with a twopart dose $(0.125 \mathrm{~g} \mathrm{q} 12 \mathrm{~h})$ was required to achieve the target CFR at $93.30 \%$, whereas $0.25 \mathrm{~g}$ infusion over $24 \mathrm{hr}$ achieved CFR at only $81.31 \%$. However, the 3-hour prolonged infusion provided a CFR of $90 \%$ for all dosing regimens regardless of renal function. In addition to prolonged infusion, frequent dosing was also found to provide a higher likelihood of bactericidal exposure than a high total daily dose. In patients receiving vasopressors, all simulated dosing regimens achieved a CFR of $90 \%$, except for the 30-minute infusion of $0.5 \mathrm{~g} \mathrm{q} 12$ hours at CLcr $\geq 81 \mathrm{~mL} / \mathrm{min}, 0.25 \mathrm{~g} \mathrm{q} 12$ hours at CLcr 51-80 mL/min, and $0.5 \mathrm{~g} \mathrm{q} 24$ hours at CLcr $26-50 \mathrm{~mL} / \mathrm{min}$. A CFR of $>90 \%$ was achieved with a 3-hour prolonged infusion of these regimens. Based on the results of PTA and CFR analysis, we made recommendations for both groups of patients (with or without vasopressor) with different degrees of renal function according to MIC $2 \mu \mathrm{g} / \mathrm{mL}$ (Table 4), MIC $1 \mu \mathrm{g} / \mathrm{mL}$ (Table 5) and $\mathrm{MIC}_{90} 0.25 \mu \mathrm{g} / \mathrm{mL}$ (Table 6). 
Table I Antimicrobial Dosing Regimens for Simulation

\begin{tabular}{|c|c|c|c|c|c|}
\hline Renal Function CLcr (mL/min) & Total Daily Dose & Dosage Regimens & Infusion Time & Non-Vasopressor Receiver & Vasopressor Receiver \\
\hline \multirow[t]{14}{*}{$81-100$} & \multirow[t]{2}{*}{$6 \mathrm{~g}$} & \multirow[t]{2}{*}{$2 \mathrm{~g} q 8 \mathrm{hr}$} & $30 \mathrm{~min}^{\mathrm{a}}$ & l & I \\
\hline & & & $3 h r^{d}$ & I & l \\
\hline & \multirow[t]{2}{*}{$4 g$} & \multirow[t]{2}{*}{$\lg 96 \mathrm{hr}$} & $30 \min ^{e}$ & 1 & 1 \\
\hline & & & $3 h r^{e}$ & I & I \\
\hline & \multirow[t]{2}{*}{$3 g$} & \multirow[t]{2}{*}{$\lg$ q $8 \mathrm{hr}$} & $30 \min ^{\mathrm{b}}$ & l & 1 \\
\hline & & & $3 h r^{e}$ & I & l \\
\hline & \multirow[t]{2}{*}{$2 g$} & \multirow[t]{2}{*}{$0.5 \mathrm{~g} \mathrm{q} 6 \mathrm{hr}$} & $30 \min ^{\mathrm{e}}$ & I & I \\
\hline & & & $3 \mathrm{hr} \mathrm{e}^{\mathrm{e}}$ & I & l \\
\hline & \multirow[t]{2}{*}{$1.5 \mathrm{~g}$} & \multirow[t]{2}{*}{$0.5 \mathrm{~g} q 8 \mathrm{hr}$} & $30 \mathrm{~min}^{\mathrm{c}}$ & I & l \\
\hline & & & $3 \mathrm{hr} \mathrm{e}^{\mathrm{e}}$ & I & I \\
\hline & \multirow[t]{2}{*}{$\lg$} & \multirow[t]{2}{*}{$0.5 \mathrm{~g}$ q $12 \mathrm{hr}$} & $30 \min ^{\mathrm{e}}$ & - & l \\
\hline & & & $3 h r^{e}$ & - & I \\
\hline & \multirow[t]{2}{*}{$0.75 \mathrm{~g}$} & \multirow[t]{2}{*}{$0.25 \mathrm{~g} \mathrm{q} 8 \mathrm{hr}$} & $30 \min ^{e}$ & - & I \\
\hline & & & $3 \mathrm{hr} \mathrm{e}^{\mathrm{e}}$ & - & I \\
\hline \multirow[t]{18}{*}{$51-80$} & \multirow[t]{2}{*}{$6 \mathrm{~g}$} & \multirow[t]{2}{*}{$2 \mathrm{~g} q 8 \mathrm{hr}$} & $30 \mathrm{~min}^{\mathrm{a}}$ & I & I \\
\hline & & & $3 \mathrm{hr} \mathrm{r}^{\mathrm{d}}$ & I & I \\
\hline & \multirow[t]{2}{*}{$4 \mathrm{~g}$} & \multirow[t]{2}{*}{$\lg 96 \mathrm{hr}$} & $30 \min ^{e}$ & I & - \\
\hline & & & $3 \mathrm{hr} \mathrm{r}^{\mathrm{e}}$ & I & - \\
\hline & \multirow[t]{2}{*}{$3 g$} & \multirow[t]{2}{*}{$\lg$ q $8 \mathrm{hr}$} & $30 \min ^{\mathrm{b}}$ & 1 & 1 \\
\hline & & & $3 h r^{e}$ & I & I \\
\hline & \multirow[t]{2}{*}{$2 g$} & \multirow[t]{2}{*}{$0.5 \mathrm{~g} \mathrm{q} 6 \mathrm{hr}$} & $30 \min ^{e}$ & I & - \\
\hline & & & $3 h r^{e}$ & I & - \\
\hline & \multirow[t]{2}{*}{$1.5 \mathrm{~g}$} & \multirow[t]{2}{*}{$0.5 \mathrm{~g} q 8 \mathrm{hr}$} & $30 \mathrm{~min}^{\mathrm{c}}$ & I & I \\
\hline & & & $3 h r^{e}$ & I & I \\
\hline & \multirow[t]{2}{*}{$\lg$} & \multirow[t]{2}{*}{$0.25 \mathrm{~g} \mathrm{q} 6 \mathrm{hr}$} & $30 \min ^{e}$ & I & - \\
\hline & & & $3 \mathrm{hr}^{\mathrm{e}}$ & I & - \\
\hline & \multirow[t]{2}{*}{$0.5 \mathrm{~g}$} & \multirow[t]{2}{*}{$0.5 \mathrm{~g} \mathrm{q} 12 \mathrm{hr}$} & $30 \min ^{e}$ & - & I \\
\hline & & & $3 \mathrm{hr}^{\mathrm{e}}$ & - & I \\
\hline & $0.75 \mathrm{~g}$ & $0.25 \mathrm{~g} \mathrm{q} 8 \mathrm{hr}$ & $30 \min ^{e}$ & I & - \\
\hline & & & $3 \mathrm{hr} \mathrm{e}^{\mathrm{e}}$ & I & - \\
\hline & $0.5 \mathrm{~g}$ & $0.25 \mathrm{~g} q 12 \mathrm{hr}$ & $30 \min ^{\mathrm{e}}$ & - & I \\
\hline & & & $3 \mathrm{hr} \mathrm{r}^{\mathrm{e}}$ & - & I \\
\hline
\end{tabular}

(Continued) 
Table I (Continued).

\begin{tabular}{|c|c|c|c|c|c|}
\hline Renal Function CLcr (mL/min) & Total Daily Dose & Dosage Regimens & Infusion Time & Non-Vasopressor Receiver & Vasopressor Receiver \\
\hline \multirow[t]{18}{*}{$26-50$} & \multirow[t]{2}{*}{$4 g$} & \multirow[t]{2}{*}{$2 \mathrm{~g} q 12 \mathrm{hr}$} & $30 \min ^{\mathrm{a}}$ & I & l \\
\hline & & & $3 \mathrm{hr}^{\mathrm{e}}$ & I & l \\
\hline & \multirow[t]{2}{*}{$3 g$} & \multirow[t]{2}{*}{$\lg$ q $8 \mathrm{hr}$} & $30 \mathrm{~min}^{\mathrm{d}}$ & I & l \\
\hline & & & $3 \mathrm{hr}^{\mathrm{e}}$ & I & - \\
\hline & \multirow[t]{2}{*}{$2 g$} & \multirow[t]{2}{*}{$\lg q 12 \mathrm{hr}$} & $30 \min ^{\mathrm{b}}$ & I & l \\
\hline & & & $3 \mathrm{hr}^{\mathrm{e}}$ & I & 1 \\
\hline & \multirow[t]{2}{*}{$1.5 \mathrm{~g}$} & \multirow[t]{2}{*}{$0.5 \mathrm{~g} q 8 \mathrm{hr}$} & $30 \min ^{\mathrm{e}}$ & I & - \\
\hline & & & $3 \mathrm{hr}^{\mathrm{e}}$ & l & - \\
\hline & \multirow[t]{2}{*}{$\lg$} & \multirow[t]{2}{*}{$0.5 \mathrm{~g} \mathrm{q} 12 \mathrm{hr}$} & $30 \min ^{c}$ & I & l \\
\hline & & & $3 \mathrm{hr}^{\mathrm{e}}$ & I & I \\
\hline & \multirow[t]{2}{*}{$0.75 \mathrm{~g}$} & \multirow[t]{2}{*}{$0.25 \mathrm{~g} \mathrm{q} 8 \mathrm{hr}$} & $30 \min ^{\mathrm{e}}$ & I & - \\
\hline & & & $3 \mathrm{hr}^{\mathrm{e}}$ & I & - \\
\hline & \multirow[t]{4}{*}{$0.5 \mathrm{~g}$} & \multirow[t]{2}{*}{$0.25 \mathrm{~g} \mathrm{q} 12 \mathrm{hr}$} & $30 \min ^{\mathrm{e}}$ & I & l \\
\hline & & & $3 \mathrm{hr}^{\mathrm{e}}$ & I & I \\
\hline & & \multirow[t]{2}{*}{$0.5 \mathrm{~g}$ q $24 \mathrm{hr}$} & $30 \min ^{\mathrm{e}}$ & - & l \\
\hline & & & $3 \mathrm{hr}^{\mathrm{e}}$ & - & l \\
\hline & \multirow[t]{2}{*}{$0.25 \mathrm{~g}$} & \multirow[t]{2}{*}{$0.125 \mathrm{~g}$ q $12 \mathrm{hr}$} & $30 \min ^{e}$ & - & I \\
\hline & & & $3 \mathrm{hr}^{\mathrm{e}}$ & - & I \\
\hline \multirow[t]{14}{*}{$11-25$} & \multirow[t]{2}{*}{$2 g$} & \multirow[t]{2}{*}{$\lg q 12 \mathrm{hr}$} & $30 \mathrm{~min}^{\mathrm{a}, \mathrm{d}}$ & I & I \\
\hline & & & $3 \mathrm{hr} r^{\mathrm{e}}$ & 1 & - \\
\hline & \multirow[t]{2}{*}{$\lg$} & \multirow[t]{2}{*}{$0.5 \mathrm{~g}$ q $12 \mathrm{hr}$} & $30 \min ^{\mathrm{b}}$ & I & I \\
\hline & & & $3 \mathrm{hr}^{\mathrm{e}}$ & I & 1 \\
\hline & \multirow[t]{4}{*}{$0.5 \mathrm{~g}$} & \multirow[t]{2}{*}{$0.25 \mathrm{~g} \mathrm{q} 12 \mathrm{hr}$} & $30 \min ^{c}$ & I & 1 \\
\hline & & & $3 \mathrm{hr}^{\mathrm{e}}$ & I & I \\
\hline & & \multirow[t]{2}{*}{$0.5 \mathrm{~g}$ q $24 \mathrm{hr}$} & $30 \min ^{\mathrm{e}}$ & - & I \\
\hline & & & $3 \mathrm{hr}^{\mathrm{e}}$ & - & 1 \\
\hline & \multirow[t]{4}{*}{$0.25 \mathrm{~g}$} & \multirow[t]{2}{*}{$0.125 \mathrm{~g}$ q $12 \mathrm{hr}$} & $30 \min ^{e}$ & I & I \\
\hline & & & $3 \mathrm{hr}^{\mathrm{e}}$ & I & I \\
\hline & & \multirow[t]{2}{*}{$0.25 \mathrm{~g}$ q $24 \mathrm{hr}$} & $30 \min ^{e}$ & - & I \\
\hline & & & $3 \mathrm{hr}^{\mathrm{e}}$ & - & I \\
\hline & \multirow[t]{2}{*}{$0.125 \mathrm{~g}$} & $0.125 \mathrm{~g}$ q $24 \mathrm{hr}$ & $30 \min ^{e}$ & - & I \\
\hline & & & $3 \mathrm{hr}^{\mathrm{e}}$ & - & I \\
\hline
\end{tabular}

(Continued) 
Table I (Continued).

\begin{tabular}{|c|c|c|c|c|c|}
\hline Renal Function CLcr (mL/min) & Total Daily Dose & Dosage Regimens & Infusion Time & Non-Vasopressor Receiver & Vasopressor Receiver \\
\hline \multirow[t]{8}{*}{$1-10$} & $2 g$ & $\lg q 12 \mathrm{hr}$ & $30 \min ^{d}$ & l & l \\
\hline & \multirow[t]{2}{*}{$\lg$} & $\lg q 24 \mathrm{hr}$ & $30 \mathrm{~min}^{\mathrm{a}}$ & l & l \\
\hline & & $0.5 \mathrm{~g} \mathrm{q} 12 \mathrm{hr}$ & $30 \min ^{\mathrm{e}}$ & I & - \\
\hline & \multirow[t]{2}{*}{$0.5 \mathrm{~g}$} & $0.5 \mathrm{~g}$ q $24 \mathrm{hr}$ & $30 \mathrm{~min}^{\mathrm{b}}$ & I & l \\
\hline & & $0.25 \mathrm{~g} q 12 \mathrm{hr}$ & $30 \min ^{\mathrm{e}}$ & l & - \\
\hline & \multirow[t]{2}{*}{$0.25 \mathrm{~g}$} & $0.25 \mathrm{~g} q 24 \mathrm{hr}$ & $30 \min ^{\mathrm{c}}$ & l & l \\
\hline & & $0.125 \mathrm{~g} \mathrm{q} 12 \mathrm{hr}$ & $30 \min ^{\mathrm{e}}$ & I & I \\
\hline & $0.125 \mathrm{~g}$ & $0.125 \mathrm{~g} \mathrm{q} 24 \mathrm{hr}$ & $30 \mathrm{~min}^{\mathrm{e}}$ & - & I \\
\hline
\end{tabular}

Notes: ${ }^{\mathrm{a} E M A}$ recommended high-dose regimen. ${ }^{\mathrm{b} U S-F D A}$ recommended high-dose regimens. ${ }^{\mathrm{C}}$ US-FDA recommended low-dose regimens. ${ }^{\mathrm{d}}$ Ehmann et al study regimens at CLSI breakpoint. ${ }^{e}$ Our study regimens.

Abbreviations: CLcr, creatinine clearance; q 6hr, every 6 hours; q $8 \mathrm{hr}$, every 8 hours; q $12 \mathrm{hr}$, every 12 hours; q $24 \mathrm{hr}$, every 24 hours.

Table 2 Susceptibility Pattern of Antimicrobial Agents for ESBL E. coli

\begin{tabular}{|c|c|c|c|}
\hline Antibiotics & Sensitive Isolates (n) & MIC Range ( $\mu \mathrm{g} / \mathrm{mL})$ & $\mathbf{S} \%$ \\
\hline Ampicillin & 0 & 32 & 0 \\
\hline Aztreonam & 20 & $2-64$ & 21.5 \\
\hline Cefazolin & 0 & 4-64 & 0 \\
\hline Ceftriaxone & 6 & $1-64$ & 6.5 \\
\hline Cefepime & 29 & $1-64$ & 31.2 \\
\hline Amikacin & 90 & $1-64$ & 96.8 \\
\hline Gentamycin & 40 & $1-16$ & 43 \\
\hline Tetracycline & 14 & $1-16$ & 15.1 \\
\hline Trimethoprim-Sulfamethoxazole & 0 & $20-320$ & 0 \\
\hline Levofloxacin & 5 & $0.12-18$ & 5.4 \\
\hline Ciprofloxacin & 10 & $0.25-4$ & 10.8 \\
\hline Amoxicillin/Clavulanate & 25 & $2-32$ & 26.9 \\
\hline Ampicillin/Sulbactam & 8 & $4-32$ & 8.6 \\
\hline Piperacillin/Tazobactam & 70 & $4-128$ & 75.3 \\
\hline Imipenem & 93 & $0.25-0.5$ & 100 \\
\hline Meropenem & 93 & $0.25-1$ & 100 \\
\hline Ertapenem & 93 & $0.12-0.5$ & 100 \\
\hline
\end{tabular}

Abbreviations: MIC, minimum inhibitory concentration; S, sensitivity; \%, percentage.

\section{Discussion}

In our study, 24.38\% was found as ESBL-producing E. coli. However, the prevalence finding was lower than recent studies in Yangon, Myanmar by Aung et al with 38\% and 49.1\% was reported by Myat et al. ${ }^{6,7}$ Both studies showed that the positive rates of ESBL genes were highest in blood isolates, while our study showed that more than half of ESBL 


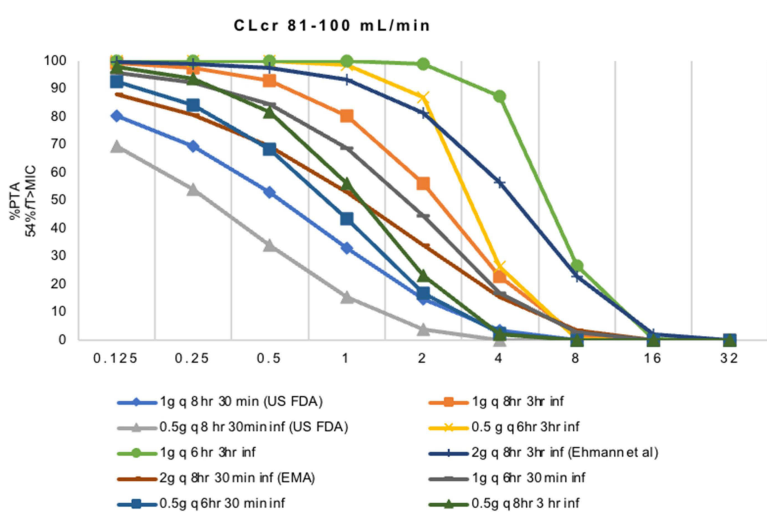

A

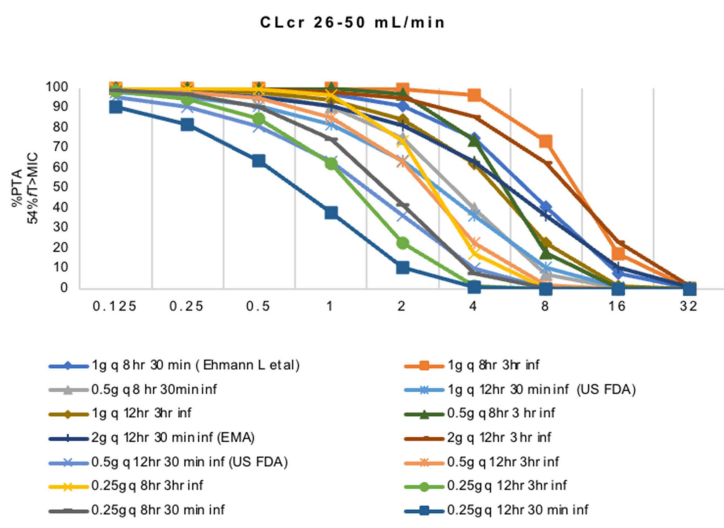

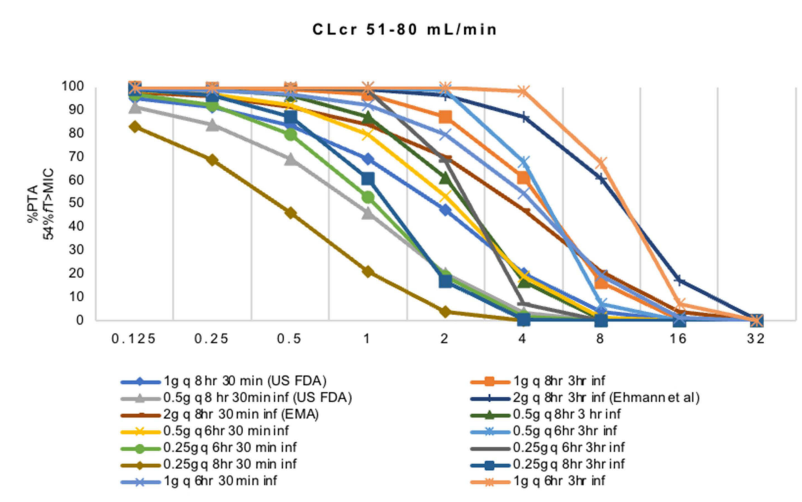

B

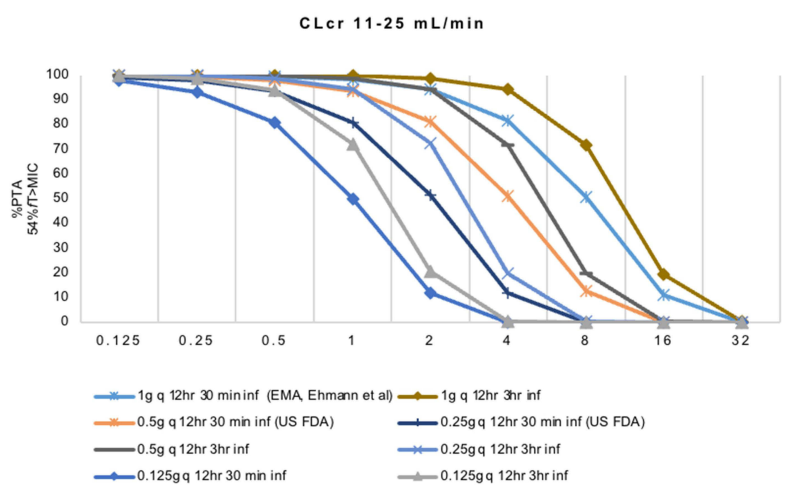

C

D

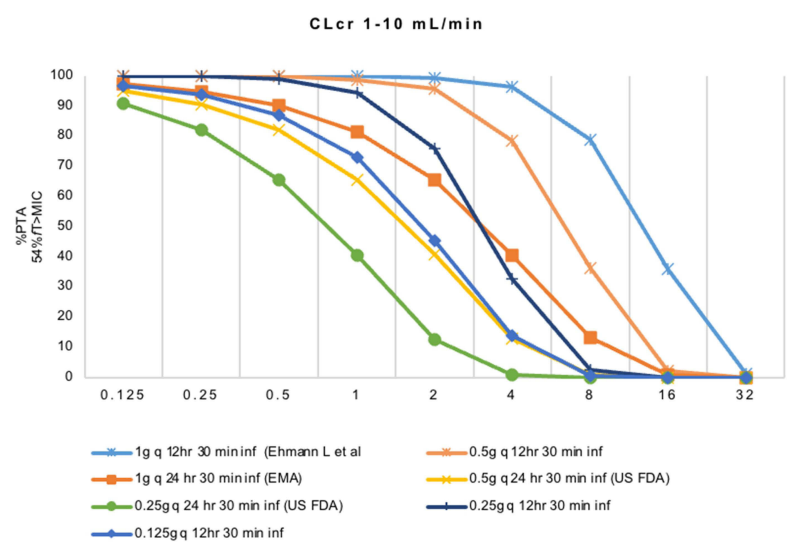

E

Figure I Probability of target attainment (PTA) of meropenem dosing regimens in patients who do not receive vasopressors and various degrees of renal functions. (A) CLcr $81-100 \mathrm{~mL} / \mathrm{min}$, (B) CLcr $51-80 \mathrm{~mL} / \mathrm{min}$, (C) CLcr 26-50 mL/min, (D) CLcr II-25 mL/min, (E) CLcr I-10 mL/min.

Abbreviations: US-FDA, The United States Food and Drug Administration; EMA, European Medicines Agency; CLcr, creatinine clearance; MIC, minimum inhibitory concentration; inf, infusion; q 6hr, every 6 hours; q $8 \mathrm{hr}$, every 8 hours; q $12 \mathrm{hr}$, every 12 hours; q 24hr, every 24 hours.

E. coli were mainly from urine samples. Myat et al investigated in three major hospitals of Yangon and retrospectively collected the clinical isolates only from blood samples of bacteremia patients, which indicated the high prevalence of ESBL E. coli and the high positive rate in blood samples. That could be due to a higher prevalence of multidrug-resistant 
CLcr $81-100 \mathrm{~mL} / \mathrm{min}$
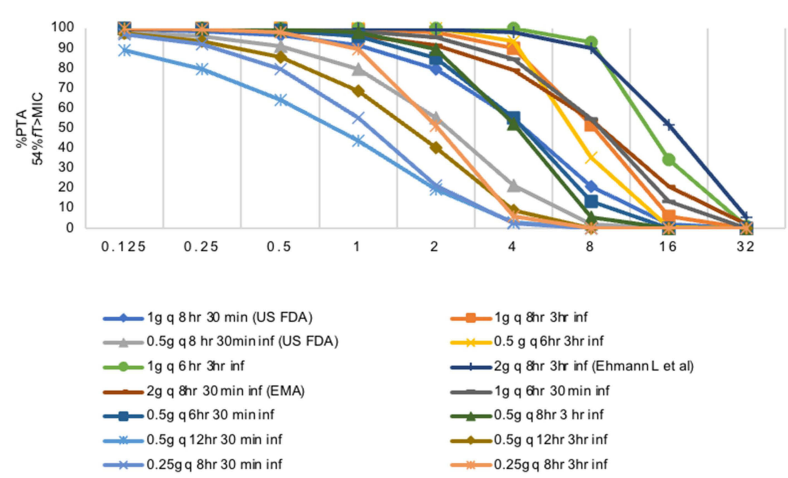

A

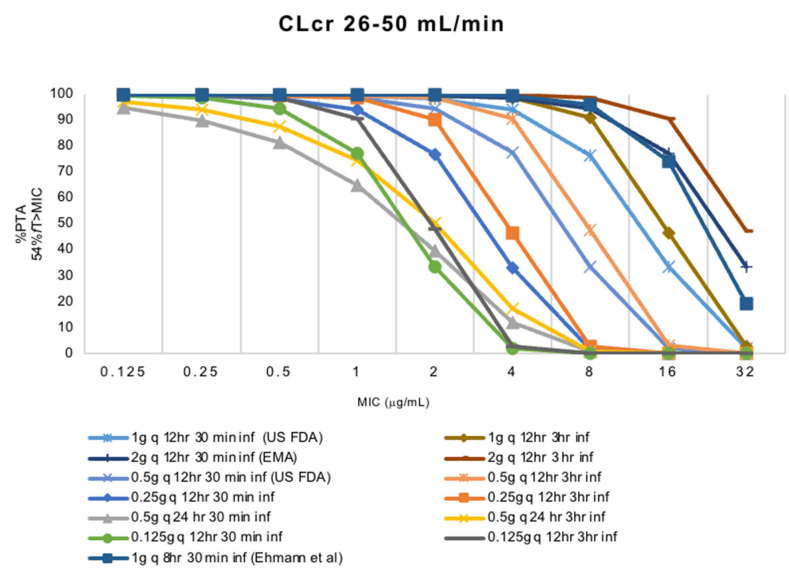

C
CLcr $51-80 \mathrm{~mL} / \mathrm{min}$
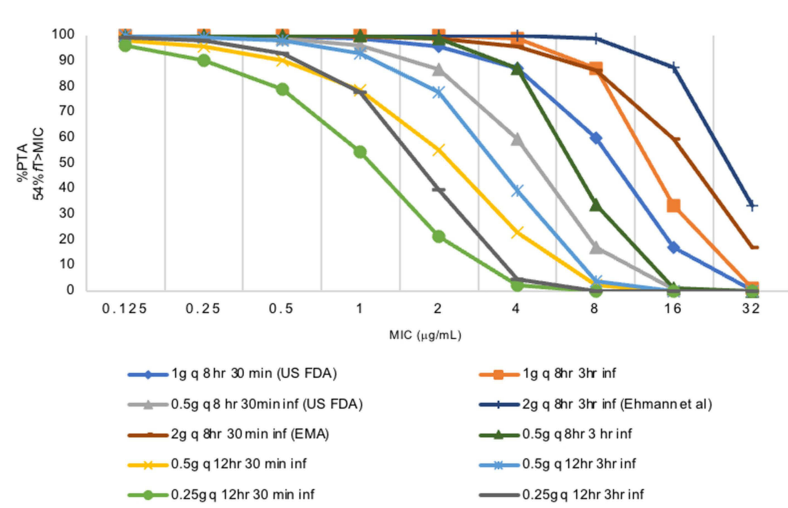

B

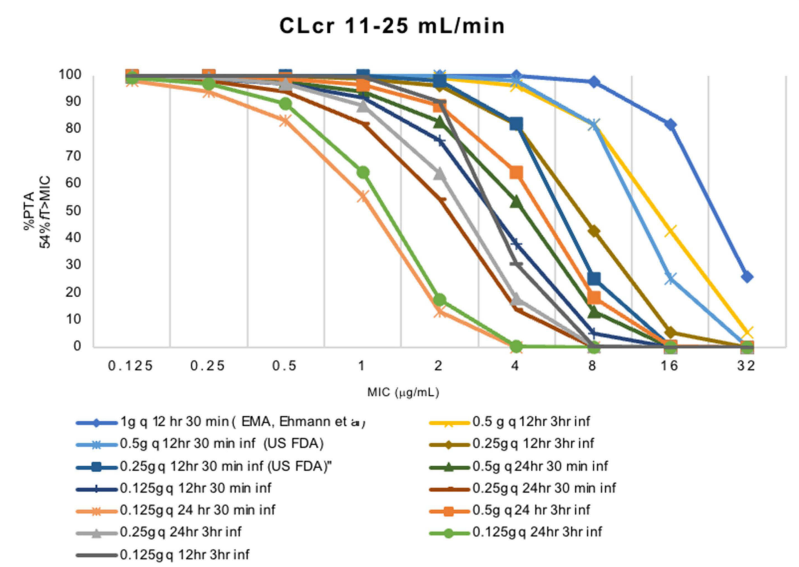

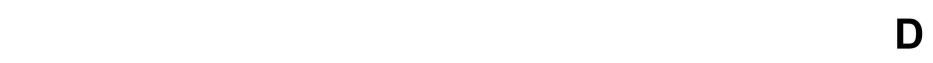

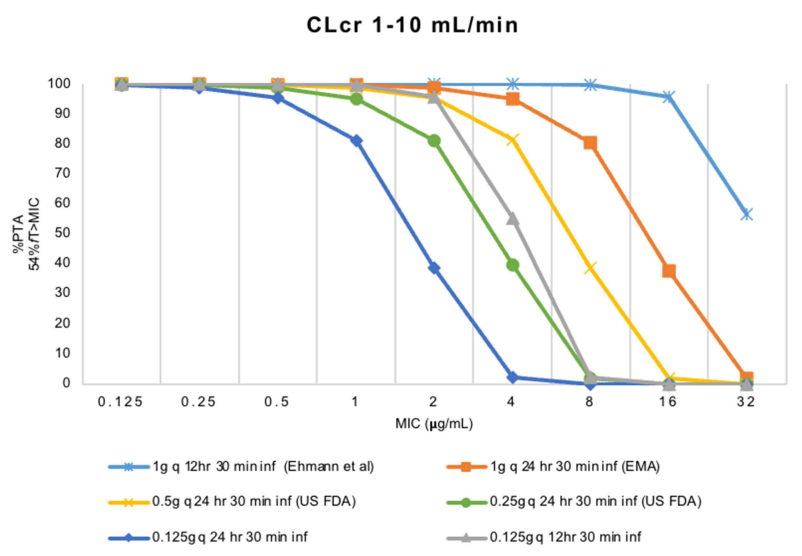

E

Figure 2 Probability of target attainment (PTA) of meropenem dosing regimens in patients who receive vasopressors and various degrees of renal functions. (A) CLcr $8 \mathrm{I}-$ $100 \mathrm{~mL} / \mathrm{min}$, (B) CLcr $51-80 \mathrm{~mL} / \mathrm{min}$, (C) CLcr 26-50 mL/min, (D) CLcr II-25 mL/min, (E) CLcr I-10 mL/min.

Abbreviations: US-FDA, The United States Food and Drug Administration; EMA, European Medicines Agency; CLcr, creatinine clearance; MIC, minimum inhibitory concentration; inf, infusion; q $6 \mathrm{hr}$, every 6 hours; q $8 \mathrm{hr}$, every 8 hours; q $12 \mathrm{hr}$, every 12 hours; q $24 \mathrm{hr}$, every 24 hours. 
Table 3 Cumulative Fraction Response (CFR) Results for All Simulated Meropenem Dosage Regimens Against ESBL E. coli

\begin{tabular}{|c|c|c|c|c|c|}
\hline \multirow{2}{*}{$\begin{array}{l}\text { Renal Function CLcr } \\
\text { (mL/min) }\end{array}$} & \multirow[t]{2}{*}{ Total Daily Dose } & \multirow[t]{2}{*}{ Regimens } & \multirow[t]{2}{*}{ Infusion Time } & \multicolumn{2}{|c|}{ CFR } \\
\hline & & & & $\begin{array}{l}\text { Non-Vasopressor } \\
\text { Receiver }\end{array}$ & Vasopressor Receiver \\
\hline \multirow[t]{14}{*}{$81-100$} & \multirow[t]{2}{*}{$6 g$} & \multirow[t]{2}{*}{$2 \mathrm{~g} \mathrm{q} 8 \mathrm{hr}$} & $30 \min ^{\mathrm{a}}$ & 80.45 & 99.41 \\
\hline & & & $3 \mathrm{hr}{ }^{\mathrm{d}}$ & 99.08 & 99.98 \\
\hline & \multirow[t]{2}{*}{$4 g$} & \multirow[t]{2}{*}{$\lg 96 \mathrm{hr}$} & $30 \min ^{e}$ & 91.99 & 99.89 \\
\hline & & & $3 \mathrm{hr} \mathrm{e}^{\mathrm{e}}$ & 99.99 & 99.99 \\
\hline & \multirow[t]{2}{*}{$3 g$} & \multirow[t]{2}{*}{ Ig q $8 \mathrm{hr}$} & $30 \min ^{b}$ & 68.86 & 98.40 \\
\hline & & & $3 \mathrm{hr}^{\mathrm{e}}$ & 97.53 & 99.96 \\
\hline & \multirow[t]{2}{*}{$2 g$} & \multirow[t]{2}{*}{$0.5 \mathrm{~g} \mathrm{q} 6 \mathrm{hr}$} & $30 \mathrm{~min}$ & 83.77 & 99.66 \\
\hline & & & $3 \mathrm{hr}^{\mathrm{e}}$ & 99.98 & 99.99 \\
\hline & \multirow[t]{2}{*}{$1.5 \mathrm{~g}$} & \multirow[t]{2}{*}{$0.5 \mathrm{~g} \mathrm{q} 8 \mathrm{hr}$} & $30 \mathrm{~min}^{\mathrm{c}}$ & 53.40 & 95.95 \\
\hline & & & $3 \mathrm{hr} \mathrm{e}^{\mathrm{e}}$ & 93.09 & 99.91 \\
\hline & \multirow[t]{2}{*}{$\lg$} & \multirow[t]{2}{*}{$0.5 \mathrm{~g} \mathrm{q} 12 \mathrm{hr}$} & $30 \min ^{e}$ & - & 79.17 \\
\hline & & & $3 \mathrm{hr}^{\mathrm{e}}$ & - & 93.47 \\
\hline & \multirow[t]{2}{*}{$0.75 g$} & \multirow[t]{2}{*}{$0.25 \mathrm{~g} \mathrm{q} 8 \mathrm{hr}$} & $30 \min ^{\mathrm{e}}$ & - & 91.68 \\
\hline & & & $3 \mathrm{hr}^{\mathrm{e}}$ & - & 99.53 \\
\hline \multirow[t]{18}{*}{$51-80$} & \multirow[t]{2}{*}{$6 g$} & \multirow[t]{2}{*}{$2 \mathrm{~g} \mathrm{q} 8 \mathrm{hr}$} & $30 \min ^{\mathrm{a}}$ & 95.86 & 99.96 \\
\hline & & & $3 \mathrm{hr}{ }^{\mathrm{d}}$ & 99.95 & 99.99 \\
\hline & \multirow[t]{2}{*}{$4 g$} & \multirow[t]{2}{*}{$\lg 96 \mathrm{hr}$} & $30 \min ^{\mathrm{e}}$ & 98.86 & - \\
\hline & & & $3 \mathrm{hr}^{\mathrm{e}}$ & 99.99 & - \\
\hline & \multirow[t]{2}{*}{$3 g$} & \multirow[t]{2}{*}{$\lg q 8 \mathrm{hr}$} & $30 \min ^{\mathrm{b}}$ & 91.10 & 99.83 \\
\hline & & & $3 \mathrm{hr}{ }^{\mathrm{e}}$ & 99.71 & 99.99 \\
\hline & \multirow[t]{2}{*}{$2 g$} & \multirow[t]{2}{*}{$0.5 \mathrm{~g} \mathrm{q} 6 \mathrm{hr}$} & $30 \min ^{\mathrm{e}}$ & 96.91 & - \\
\hline & & & $3 \mathrm{hr} \mathrm{e}^{\mathrm{e}}$ & 99.99 & - \\
\hline & $1.5 \mathrm{~g}$ & $0.5 \mathrm{~g} \mathrm{q} 8 \mathrm{hr}$ & $30 \mathrm{~min}^{\mathrm{c}}$ & 83.61 & 99.63 \\
\hline & & & $3 \mathrm{hr}^{\mathrm{e}}$ & 98.99 & 99.99 \\
\hline & $\lg$ & $0.25 \mathrm{~g} \mathrm{q} 6 \mathrm{hr}$ & $30 \min ^{e}$ & 91.92 & - \\
\hline & & & $3 \mathrm{hr} \mathrm{e}^{\mathrm{e}}$ & 99.98 & - \\
\hline & $0.5 \mathrm{~g}$ & $0.5 \mathrm{~g} \mathrm{q} 12 \mathrm{hr}$ & $30 \min ^{e}$ & - & 95.47 \\
\hline & & & $3 \mathrm{hr}{ }^{\mathrm{e}}$ & - & 99.29 \\
\hline & $0.75 \mathrm{~g}$ & $0.25 \mathrm{~g} \mathrm{q} 8 \mathrm{hr}$ & $30 \min ^{e}$ & 68.32 & - \\
\hline & & & $3 \mathrm{hr}{ }^{\mathrm{e}}$ & 96.18 & - \\
\hline & $0.5 g$ & $0.25 \mathrm{~g}$ q I $2 \mathrm{hr}$ & $30 \min ^{e}$ & - & 89.78 \\
\hline & & & $3 \mathrm{hr} \mathrm{e}^{\mathrm{e}}$ & - & 97.77 \\
\hline
\end{tabular}


Table 3 (Continued).

\begin{tabular}{|c|c|c|c|c|c|}
\hline \multirow{2}{*}{$\begin{array}{l}\text { Renal Function CLcr } \\
\text { (mL/min) }\end{array}$} & \multirow{2}{*}{ Total Daily Dose } & \multirow{2}{*}{ Regimens } & \multirow{2}{*}{ Infusion Time } & \multicolumn{2}{|c|}{ CFR } \\
\hline & & & & $\begin{array}{l}\text { Non-Vasopressor } \\
\text { Receiver }\end{array}$ & Vasopressor Receiver \\
\hline \multirow[t]{12}{*}{$26-50$} & \multirow[t]{2}{*}{$4 g$} & \multirow[t]{2}{*}{$2 \mathrm{~g} \mathrm{q} 12 \mathrm{hr}$} & $30 \mathrm{~min}^{\mathrm{a}}$ & 97.60 & 99.98 \\
\hline & & & $3 \mathrm{hr}^{\mathrm{e}}$ & 99.69 & 99.99 \\
\hline & \multirow[t]{2}{*}{$3 g$} & \multirow[t]{2}{*}{$\lg q 8 \mathrm{hr}$} & $30 \mathrm{~min}^{\mathrm{d}}$ & 99.63 & 99.99 \\
\hline & & & $3 \mathrm{hr}^{\mathrm{e}}$ & 99.99 & - \\
\hline & \multirow[t]{2}{*}{$2 g$} & \multirow[t]{2}{*}{$\lg q 12 \mathrm{hr}$} & $30 \mathrm{~min}^{\mathrm{b}}$ & 95.25 & 99.93 \\
\hline & & & $3 \mathrm{hr} \mathrm{e}^{\mathrm{e}}$ & 99.23 & 99.98 \\
\hline & \multirow[t]{2}{*}{$1.5 \mathrm{~g}$} & \multirow[t]{2}{*}{$0.5 \mathrm{~g} \mathrm{q} 8 \mathrm{hr}$} & $30 \min ^{\mathrm{e}}$ & 98.86 & - \\
\hline & & & $3 h r^{e}$ & 99.98 & - \\
\hline & \multirow[t]{2}{*}{$\lg$} & \multirow[t]{2}{*}{$0.5 \mathrm{~g} \mathrm{q} \mathrm{I2hr}$} & $30 \min ^{c}$ & 90.22 & 99.87 \\
\hline & & & $3 \mathrm{hr} \mathrm{e}^{\mathrm{e}}$ & 97.89 & 99.99 \\
\hline & \multirow[t]{2}{*}{$0.75 \mathrm{~g}$} & \multirow[t]{2}{*}{$0.25 \mathrm{~g} \mathrm{q} 8 \mathrm{hr}$} & $30 \min ^{\mathrm{e}}$ & 96.68 & - \\
\hline & & & $3 \mathrm{hr} \mathrm{e}^{\mathrm{e}}$ & 99.85 & - \\
\hline \multirow[t]{14}{*}{$11-25$} & \multirow[t]{2}{*}{$2 g$} & \multirow[t]{2}{*}{$\operatorname{lg~q~} 12 \mathrm{hr}$} & $30 \min ^{\mathrm{a}, \mathrm{d}}$ & 99.73 & 99.99 \\
\hline & & & $3 \mathrm{hr} \mathrm{e}^{\mathrm{e}}$ & 99.98 & \\
\hline & \multirow[t]{2}{*}{$\lg$} & \multirow[t]{2}{*}{$0.5 \mathrm{~g} \mathrm{q} 12 \mathrm{hr}$} & $30 \mathrm{~min}^{\mathrm{b}}$ & 99.20 & 99.99 \\
\hline & & & $3 \mathrm{hr} r^{\mathrm{e}}$ & 99.93 & 99.99 \\
\hline & \multirow[t]{4}{*}{$0.5 \mathrm{~g}$} & \multirow[t]{2}{*}{$0.25 \mathrm{~g} q / 2 \mathrm{hr}$} & $30 \min ^{\mathrm{c}}$ & 97.62 & 99.99 \\
\hline & & & $3 \mathrm{hr}{ }^{\mathrm{e}}$ & 99.66 & 99.88 \\
\hline & & \multirow[t]{2}{*}{$0.5 \mathrm{~g}$ q $24 \mathrm{hr}$} & $30 \min ^{\mathrm{e}}$ & - & 99.09 \\
\hline & & & $3 \mathrm{hr}^{\mathrm{e}}$ & - & 99.60 \\
\hline & \multirow[t]{4}{*}{$0.25 \mathrm{~g}$} & \multirow{2}{*}{$\begin{array}{l}0.125 \mathrm{~g} \\
q 12 \mathrm{hr}\end{array}$} & $30 \min ^{\mathrm{e}}$ & 92.60 & 98.69 \\
\hline & & & $3 \mathrm{hr} \mathrm{e}^{\mathrm{e}}$ & 98.34 & 99.99 \\
\hline & & \multirow[t]{2}{*}{$0.25 \mathrm{~g} q 24 \mathrm{hr}$} & $30 \min ^{\mathrm{e}}$ & - & 97.63 \\
\hline & & & $3 h r^{e}$ & - & 98.91 \\
\hline & \multirow[t]{2}{*}{$0.125 \mathrm{~g}$} & \multirow{2}{*}{$\begin{array}{l}0.125 \mathrm{~g} \\
q 24 \mathrm{hr}\end{array}$} & $30 \mathrm{~min}^{\mathrm{e}}$ & - & 93.59 \\
\hline & & & $3 \mathrm{hr} \mathrm{e}^{\mathrm{s}}$ & - & 96.50 \\
\hline
\end{tabular}


Table 3 (Continued).

\begin{tabular}{|c|c|c|c|c|c|}
\hline \multirow{2}{*}{$\begin{array}{l}\text { Renal Function CLcr } \\
\text { (mL/min) }\end{array}$} & \multirow[t]{2}{*}{ Total Daily Dose } & \multirow[t]{2}{*}{ Regimens } & \multirow[t]{2}{*}{ Infusion Time } & \multicolumn{2}{|c|}{ CFR } \\
\hline & & & & $\begin{array}{l}\text { Non-Vasopressor } \\
\text { Receiver }\end{array}$ & Vasopressor Receiver \\
\hline \multirow[t]{8}{*}{$1-10$} & $2 g$ & $\lg \mathrm{q} \mid 2 \mathrm{hr}$ & $30 \min ^{d}$ & 99.99 & 99.99 \\
\hline & \multirow[t]{2}{*}{$\lg$} & $\lg q 24 \mathrm{hr}$ & $30 \mathrm{~min}^{\mathrm{a}}$ & 94.63 & 99.97 \\
\hline & & $0.5 \mathrm{~g} \mathrm{q} 12 \mathrm{hr}$ & $30 \min ^{\mathrm{e}}$ & 99.98 & - \\
\hline & \multirow[t]{2}{*}{$0.5 \mathrm{~g}$} & $0.5 \mathrm{~g}$ q $24 \mathrm{hr}$ & $30 \min ^{\mathrm{b}}$ & 90.26 & 99.89 \\
\hline & & $0.25 \mathrm{~g} \mathrm{q} 12 \mathrm{hr}$ & $30 \min ^{\mathrm{e}}$ & 99.82 & - \\
\hline & \multirow[t]{2}{*}{$0.25 \mathrm{~g}$} & $0.25 \mathrm{~g} \mathrm{q} 24 \mathrm{hr}$ & $30 \min ^{c}$ & 81.31 & 99.55 \\
\hline & & $\begin{array}{l}0.125 \mathrm{~g} \\
q 12 \mathrm{hr}\end{array}$ & $30 \min ^{\mathrm{e}}$ & 93.30 & 99.99 \\
\hline & $0.125 \mathrm{~g}$ & $\begin{array}{l}0.125 \mathrm{~g} \\
\mathrm{q} 24 \mathrm{hr}\end{array}$ & $30 \min ^{\mathrm{e}}$ & - & 98.67 \\
\hline
\end{tabular}

Notes: ${ }^{a} E M A$ recommended high-dose regimen. ${ }^{b}$ US-FDA recommended high-dose regimens. ${ }^{\circ}$ US-FDA recommended low-dose regimens. ${ }^{d}$ Ehmann et al study regimens at CLSI breakpoint. ${ }^{e}$ Our study regimens.

Abbreviations: CLcr, creatinine clearance; q 6hr, every 6 hours; q $8 \mathrm{hr}$, every 8 hours; q $12 \mathrm{hr}$, every 12 hours; q $24 \mathrm{hr}$, every 24 hours.

Table 4 Recommendation for MIC $2 \mu \mathrm{g} / \mathrm{mL}$ (EUCAST Breakpoint)

\begin{tabular}{|c|c|c|c|c|c|c|}
\hline \multirow{2}{*}{$\begin{array}{l}\text { Renal Function CLcr } \\
\text { (mL/min) }\end{array}$} & \multicolumn{3}{|c|}{ Non-Vasopressor Receiving Patients } & \multicolumn{3}{|c|}{ Vasopressor Receiving Patients } \\
\hline & Regimens & TDD & $\begin{array}{l}\text { Alternative } \\
\text { Regimens }\end{array}$ & Regimens & TDD & $\begin{array}{l}\text { Alternative } \\
\text { Regimens }\end{array}$ \\
\hline $81-100$ & Ig q $6 \mathrm{hr}(3 \mathrm{hr})$ & $4 g$ & & $0.5 \mathrm{~g} \mathrm{q} 6 \mathrm{hr}(3 \mathrm{hr})$ & $2 g$ & \\
\hline $5 I-80$ & $0.5 \mathrm{~g} \mathrm{q} 6 \mathrm{hr}(3 \mathrm{hr})$ & $2 g$ & & $0.5 \mathrm{~g} \mathrm{q} 8 \mathrm{hr}(3 \mathrm{hr})$ & $1.5 \mathrm{~g}$ & \\
\hline $25-50$ & $0.5 \mathrm{~g} \mathrm{q} 8 \mathrm{hr}(3 \mathrm{hr})$ & $1.5 \mathrm{~g}$ & & $0.25 \mathrm{~g} \mathrm{q} 12 \mathrm{hr}(3 \mathrm{hr})$ & $0.5 \mathrm{~g}$ & \\
\hline $11-25$ & $0.5 \mathrm{~g} \mathrm{q} 12 \mathrm{hr}(3 \mathrm{hr})$ & $\lg$ & & $0.25 \mathrm{~g} \mathrm{q} 12 \mathrm{hr}(30 \mathrm{~min})$ & $0.5 \mathrm{~g}$ & \\
\hline $1-10$ & $0.5 \mathrm{~g}$ q $12 \mathrm{hr}(30 \mathrm{~min})$ & $\lg$ & & $0.125 \mathrm{~g} \mathrm{q} 12 \mathrm{hr}(30 \mathrm{~min})$ & 0.25 & $0.5 \mathrm{~g}$ q $24 \mathrm{hr}(30 \mathrm{~min})$ \\
\hline
\end{tabular}

Abbreviations: MIC, minimum inhibitory concentration; CLcr, creatinine clearance; EUCAST, European Committee on Antimicrobial Susceptibility Testing; TDD, total daily dose; q $6 \mathrm{hr}$, every 6 hours; q $8 \mathrm{hr}$, every 8 hours; q I hr, every 12 hours; q $24 \mathrm{hr}$, every 24 hours.

organism colonization, a lack of antimicrobial control, inadequate infection control methods, or healthcare-associated illnesses. Furthermore, the prevalence of organisms may vary by geography and timing of the study. The ESBL production rate in E. coli was 59.4\% in 2011, according to the reports of Regional Resistance Surveillance program susceptibility rates from 12 Asia-Pacific nations (APAC). The highest rate of ESBL E. coli was reported in Indonesian isolates with $71 \% .{ }^{18,19}$ Our clinical $E$. coli isolates came from a single private laboratory, and they could have come from a variety of diseases, particularly urinary tract infections, at numerous hospitals throughout upper Myanmar. In addition, the lack of accurate data from the laboratory could reduce the real prevalence rate of ESBL E. coli.

According to the MCS results, the PTA of Meropenem against ESBL E. coli in the targeted population was affected by five factors: vasopressor administration, specific MIC, CLcr, infusion length, and dose fractionation. The MCS data revealed that the risk of target attainment failure was increased when the CLcr got higher, especially $>80 \mathrm{~mL} / \mathrm{min}$. In addition, the larger rate of lower PTA values was observed when the MIC got higher $\geq 1 \mu \mathrm{g} / \mathrm{mL}$. 
Table 5 Recommendation for MIC I $\mu \mathrm{g} / \mathrm{mL}$ (CLSI Breakpoint)

\begin{tabular}{|c|c|c|c|c|c|c|}
\hline \multirow[t]{2}{*}{ Renal Function CLcr (mL/min) } & \multicolumn{3}{|c|}{ Non-Vasopressor Receiving Patients } & \multicolumn{3}{|c|}{ Vasopressor Receiving Patients } \\
\hline & Regimens & TDD & Alternative Regimens & Regimens & TDD & Alternative Regimens \\
\hline $81-100$ & $0.5 \mathrm{~g} q 6 \mathrm{hr}(3 \mathrm{hr})$ & $2 g$ & & $0.5 \mathrm{~g} \mathrm{q} 8 \mathrm{hr}(3 \mathrm{hr})$ & $1.5 \mathrm{~g}$ & \\
\hline $51-80$ & $0.25 \mathrm{~g} \mathrm{q} 6 \mathrm{hr}(3 \mathrm{hr})$ & $\lg$ & & $0.5 \mathrm{~g} \mathrm{q} \mathrm{I} 2 \mathrm{hr}(3 \mathrm{hr})$ & $\lg$ & \\
\hline $25-50$ & $0.25 \mathrm{~g} \mathrm{q} 8 \mathrm{hr}(3 \mathrm{hr})$ & $0.75 \mathrm{~g}$ & & $0.25 \mathrm{~g}$ q $12 \mathrm{hr}(30 \mathrm{~min})$ & $0.5 \mathrm{~g}$ & \\
\hline $11-25$ & $0.25 \mathrm{~g} q \mathrm{I} 2 \mathrm{hr}(3 \mathrm{hr})$ & $0.5 \mathrm{~g}$ & & $0.125 \mathrm{~g} \mathrm{q} 12 \mathrm{hr}(30 \mathrm{~min})$ & $0.25 \mathrm{~g}$ & $0.5 \mathrm{~g} \mathrm{q} 24 \mathrm{hr}(30 \mathrm{~min} / 3 \mathrm{hr})$ \\
\hline $1-10$ & $0.25 \mathrm{~g} \mathrm{q} 12 \mathrm{hr}(30 \mathrm{~min})$ & $0.5 \mathrm{~g}$ & & $0.125 \mathrm{~g} \mathrm{q} 12 \mathrm{hr}(30 \mathrm{~min})$ & $0.25 \mathrm{~g}$ & $0.25 \mathrm{~g} \mathrm{q} 24 \mathrm{hr}(30 \mathrm{~min})$ \\
\hline
\end{tabular}

Abbreviations: MIC, minimum inhibitory concentration; CLcr, creatinine clearance; CLSI, Clinical and Laboratory Standards Institute; TDD, total daily dose; q $6 \mathrm{hr}$, every 6 hours; q $8 \mathrm{hr}$, every 8 hours; q $12 \mathrm{hr}$, every 12 hours; q $24 \mathrm{hr}$, every 24 hours.

Table 6 Recommendation for MIC $0.25 \mu \mathrm{g} / \mathrm{mL}$ (MIC $50 / 90$ of Myanmar Population)

\begin{tabular}{|c|c|c|c|c|c|c|}
\hline \multirow[t]{2}{*}{ Renal Function CLcr (mL/min) } & \multicolumn{3}{|c|}{ Non-Vasopressor Receiving Patients } & \multicolumn{3}{|c|}{ Vasopressor Receiving Patients } \\
\hline & Regimens & TDD & Alternative Regimens & Regimens & TDD & Alternative Regimens \\
\hline $81-100$ & $0.5 \mathrm{~g} \mathrm{q} 8 \mathrm{hr}(3 \mathrm{hr})$ & $1.5 \mathrm{~g}$ & & $0.25 \mathrm{~g} \mathrm{q} 8 \mathrm{hr}(3 \mathrm{hr})$ & $\lg$ & \\
\hline $51-80$ & $0.25 \mathrm{~g} \mathrm{q} 8 \mathrm{hr}(3 \mathrm{hr})$ & $0.75 \mathrm{~g}$ & & $0.25 \mathrm{~g}$ q $12 \mathrm{hr}(30 \mathrm{~min})$ & $0.5 \mathrm{~g}$ & \\
\hline $25-50$ & $0.25 \mathrm{~g} \mathrm{q} 12 \mathrm{hr}(3 \mathrm{hr})$ & $0.5 \mathrm{~g}$ & & $0.125 \mathrm{~g} \mathrm{q} 12 \mathrm{hr}(30 \mathrm{~min})$ & $0.25 \mathrm{~g}$ & \\
\hline $11-25$ & $0.125 \mathrm{~g} \mathrm{q} 12 \mathrm{hr}(30 \mathrm{~min})$ & $0.25 \mathrm{~g}$ & & $0.125 \mathrm{~g} \mathrm{q} 24 \mathrm{hr}(30 \mathrm{~min})$ & $0.125 \mathrm{~g}$ & \\
\hline $1-10$ & $0.125 \mathrm{~g} \mathrm{q} 12 \mathrm{hr}(30 \mathrm{~min})$ & $0.25 \mathrm{~g}$ & $0.5 \mathrm{~g}$ q $24 \mathrm{hr}(30 \mathrm{~min})$ & $0.125 \mathrm{~g} \mathrm{q} 24 \mathrm{hr}(30 \mathrm{~min})$ & $0.125 \mathrm{~g}$ & \\
\hline
\end{tabular}

Abbreviations: MIC, minimum inhibitory concentration; $\mathrm{MIC}_{50 / 90}$, MIC for $50 \%$ and $90 \%$ of isolates tested; CLcr, creatinine clearance; TDD, total daily dose; $q$ $6 \mathrm{hr}$, every 6 hours; q 8hr, every 8 hours; q $12 \mathrm{hr}$, every 12 hours; q 24hr, every 24 hours.

In patients not receiving vasopressor with CLcr $>80 \mathrm{~mL} / \mathrm{min}$, the current reference regimens (US-FDA and EMA) got below $80 \%$ PTA even for MIC $0.25 \mu \mathrm{g} / \mathrm{mL}$. When the MIC was $\geq 1 \mu \mathrm{g} / \mathrm{mL}$, those regimens failed to achieve the target in almost all renal function except CLcr 11-25 mL/min whereas the regimens from Ehmann et al, study reached $>90 \%$ PTA in all CLcr ranges only except in CLcr $>80 \mathrm{~mL} / \mathrm{min}$ for MIC $2 \mu \mathrm{g} / \mathrm{mL}$. The relationship between MIC, CLcr and PTA were already supported by the previous studies. ${ }^{8,20,21}$ The maximum total daily dose of $6 \mathrm{~g} /$ day given 8 hourly $(2 \mathrm{~g}$ q $8 \mathrm{hr}$; $3 \mathrm{hr}$ infusion) could not produce $90 \%$ PTA in the subgroup of patients with CLcr $>80 \mathrm{~mL} / \mathrm{mL}$ for MIC $2 \mathrm{~g} / \mathrm{L}$, even if the PTA values rose when we simulated the 3 hour infusion regimens. In that situation, the prolonged infusion of a lower total daily dose and more fractionation ( $4 \mathrm{~g} /$ day given 6hourly) achieved up to $98.96 \%$. Mathew et al, discovered that dosage fractionation (1g q $6 \mathrm{hr}$; $3 \mathrm{hr}$ infusion, a total daily dose of $4 \mathrm{~g} /$ day) resulted in higher PTA than dosing escalation (2g q $8 \mathrm{hr}$; $3 \mathrm{hr}$ infusion, a total daily dose of $6 \mathrm{~g} /$ day) when CLcr was $>89.9 \mathrm{~mL} / \mathrm{min}^{21}$ Therefore, the combination of prolonged infusion and dosage fractionation strategy helps to improve $\% \mathrm{~T}>\mathrm{MIC}$ in patients with good renal function. Guillaume et al, authors of the French Society of Pharmacology and Therapeutic guidelines, recommend increasing the dosing frequency or infusion length while maintaining the same daily dose or escalating the unit dose by $25-50 \%$ while preserving the same frequency if the target beta-lactam concentration is not reached. ${ }^{22}$

Interestingly, in patients receiving a vasopressor, most of all reference dosing regimens achieved higher PTA and maintained $>90 \%$ at all renal functions. This could result in a lower dosing requirement compared to recipients without vasopressors. For example, at least $2 \mathrm{~g}$ /day (which was half the dosing requirement for non-vasopressor recipients) given every 6hourly $(0.5 \mathrm{~g} \mathrm{q} 4 \mathrm{hr}$ ) was sufficient to achieve the target PTA in CLcr $>80 \mathrm{~mL} / \mathrm{min}$ for MIC $2 \mu \mathrm{g} / \mathrm{mL}$ than EMA and Ehmann et al, regimens of $6 \mathrm{~g} /$ day. Apart from the lower dose requirement, another interesting point is that dosing fractionation was not necessary to achieve the target even at MIC $2 \mu \mathrm{g} / \mathrm{mL}$ in CLcr $80 \mathrm{~mL} / \mathrm{min}$ compared with patients not receiving vasopressors. 
Although there have been no MCS studies of meropenem in vasopressor receiving patients, the pharmacokinetic parameters of population PK studies and the pharmacology of vasopressor could explain the reason of the higher PTA and the lower dosage required. The vasopressor was a significant covariate, and the CL of meropenem in vasopressorreceiving patients was half that of non-vasopressor-receiving patients in Pop PK research we used. In addition, vasopressor is usually administered in critically ill patients with an unstable hemodynamic condition to prevent renal hypoperfusion when fluid resuscitation alone is not sufficient. ${ }^{23}$ Therefore, the patient who requires a vasopressor usually has worse renal function than the patient without a vasopressor. Although vasopressors bind to particular receptors and improve mean arterial pressure to $65 \mathrm{mmHg}$, they also produce localized renal vasoconstriction and ischemia, which make their influence on renal function unclear. ${ }^{24,25}$ Lankadeva et al investigated the effects of norepinephrine in septic lambs with AKI and discovered that restoring blood pressure with clinically relevant, high-dose norepinephrine aggravated the underlying renal medullary ischemia and hypoxia. ${ }^{26}$ Furthermore, vasopressors frequently cause organ ischemia, and the high-dose, rapid infusion of vasopressors causes persistent arterial hypertension, which results in a doubling of serum creatinine and the requirement for renal replacement therapy. ${ }^{27}$ As a result, though vasopressors enhance transitory renal blood flow and urinary excretion, they cannot restore renal function to normal in principle. Therefore, it should be cautious to adjust the administration of meropenem in patients receiving vasopressors.

The current high sensitivity of ESBL E. coli, with $\mathrm{MIC}_{90}$ of $0.25 \mu \mathrm{g} / \mathrm{mL}$ derived from our study's MIC distribution, enables a high microbiological success rate with 90\% CFR in most cases. Except for the 30-minute infusion, 8 hourly dosage regimens in patients not receiving vasopressors with CLcr $81 \mathrm{~mL} / \mathrm{min}$, the majority of simulated regimens achieved therapeutic effectiveness with $90 \%$ CFR. This result is similar to the study by Isla et al; in which the MIC distribution of ESBL E. coli from Europe, Spain, and the United States was quite low at $\leq 0.25 \mu \mathrm{g} / \mathrm{mL}$, and treatment success with $>90 \%$ CFR was observed with all simulated regimens except $0.5 \mathrm{~g}$ q 8-hour, 30-min infusion at CLcr $100 \mathrm{~mL} / \mathrm{min}^{20}$

Our study population has an $\mathrm{MIC}_{90}$ of $0.25 \mu \mathrm{g} / \mathrm{mL}$, and we recommend a low dose with high PTA and CFR to reduce meropenem overdose and combat antibiotic resistance. We also suggested dosing for the CLSI and EUCAST breakpoints because MIC distribution and microbiological susceptibility may vary by geography and time. For the Myanmar population with $\mathrm{MIC}_{90}$, we recommend a prolonged infusion of $0.5 \mathrm{~g}$ every 8 hours in patients not receiving vasopressors with CLcr $>80 \mathrm{~mL} / \mathrm{min}$, and $0.25 \mathrm{~g}$ every 8 hours in patients receiving vasopressors with CLcr $>80 \mathrm{~mL} / \mathrm{min}$. For CLcr $<25 \mathrm{~mL} / \mathrm{min}$, the required dosage was much lower than that recommended by the US FDA and EMA. The study by Grijalba et al showed that more than $80 \%$ of the germs (Pseudomonas aeruginosa and Escherichia coli) in their hospital were $<0.5 \mu \mathrm{g} / \mathrm{mL}$ and required a prolonged infusion of $1 \mathrm{~g} \mathrm{q} 6$ hours in patients with augmented or normal renal function (CLcr 89.5 to $140 \mathrm{~mL} / \mathrm{min}$ and 59.6 to $89.4 \mathrm{~mL} / \mathrm{min}$ ) and a prolonged infusion of $1-0.5 \mathrm{~g} \mathrm{q} 8$ hours in patients with CLcr $59.5 \mathrm{~mL} / \mathrm{min} .{ }^{28}$ Although $\mathrm{MIC}_{90}$ is quite similar to our study, the reason why the recommended doses of their study were higher is that they used a higher pharmacodynamic target of $100 \% \mathrm{~T} \mathrm{~T}>4 \mathrm{MIC}$, whereas our study used $54 \% \mathrm{f} \mathrm{T}>\mathrm{MIC}$. Therefore, the dosage of meropenem could be changed based on the pharmacodynamic target we used and the expected MIC.

Our study has some limitations. First, the meropenem susceptibility test was performed using the VITEK machine, although CLSI and EUCAST recommend broth microdilution as the standard method. However, the VITEK device is capable of accurately performing susceptibility testing and adequately interpreting the mechanism of resistance. Second, we did not have access to the confirmation and genotype of ESBL E. coli due to limited resources. Third, the clinical isolates were collected from a single private laboratory and all of these clinical isolates could have come from different infections. The true MIC may be underestimated in critically ill patients and the CFR may vary. Fourth, the PK parameters for meropenem were collected from Vietnamese critically ill patients, and those PK data may differ from patients in daily clinical practice due to variation in race, weight, renal function, disease severity, and other causes. Fifth, we did not specify the types and doses of vasopressors that might affect the PTA of meropenem because the population PK study we used did not mention the specific data about vasopressors. However, the findings of our study demonstrated that meropenem should be used with caution in vasopressor-dependent patients. Finally, our study recommended only the possible dose of meropenem to meet the PK/PD target for MIC 90 , CLSI, and EUCAST against ESBL E. coli. Despite these limitations, this study provided important information useful for the treatment of ESBL E. coli in clinical practice to combat antibiotic resistance and preserve limited antibiotics. 


\section{Conclusion}

Our study compared the meropenem dosing regimens approved by the EMA, the US-FDA, Ehmann et al, and our study against ESBL E. coli. The current US-FDA and EMA dosing regimens were inadequate for MIC of $>0.25 \mu \mathrm{g} / \mathrm{mL}$ in almost all renal function especially at CLcr of $>80 \mathrm{~mL} / \mathrm{min}$ in patients not receiving vasopressors. Dose fractionation (small dose, small intervals) combined with the prolonged infusion was found to be more effective than dose escalation when CLcr was $>50 \mathrm{~mL} / \mathrm{min}$ for high MIC $>1 \mu \mathrm{g} / \mathrm{mL}$. Lowering CLcr improved achievement of \% PTA and \%CFR. In patients with low meropenem MIC, impaired renal function, and/or vasopressor administration, less aggressive dosing strategies should be considered to balance the risks and benefits.

\section{Ethical Statement}

This study was conducted according to the guideline of the Declaration of Helsinki and approved by the institutional review board of the Defense Services Medical Research Center, Naypyidaw, Myanmar (Document No: IRB/2020/A-03), and the institutional review board, Faculty of Dentistry and Faculty of Pharmacy, Mahidol University (Document No: 78.0319/517). The study obtained the respective data from the database with the permission of the dataset owner of Right Laboratory and Health Screen, and informed consents from patients were not applicable.

\section{Acknowledgments}

We thank Right Laboratory and Health Screen for contributing the microbiological data and members of the laboratory for helping in data collection. EEW would like to thank Mahidol University for research funding from the postgraduate scholarship (Ref. No. 0517.02/04532).

\section{Disclosure}

The authors report no conflicts of interest in this work.

\section{References}

1. Paterson DL, Bonomo RA. Extended-spectrum beta-lactamases: a clinical update. Clin Microbiol Rev. 2005;18(4):657-686. doi:10.1128/ CMR.18.4.657-686.2005

2. Schwaber MJ, Carmeli Y. Mortality and delay in effective therapy associated with extended-spectrum beta-lactamase production in Enterobacteriaceae bacteraemia: a systematic review and meta-analysis. J Antimicrob Chemother. 2007;60(5):913-920. doi:10.1093/jac/dkm318

3. Rottier WC, Ammerlaan HS, Bonten MJ. Effects of confounders and intermediates on the association of bacteraemia caused by extended-spectrum $\beta$-lactamase-producing Enterobacteriaceae and patient outcome: a meta-analysis. J Antimicrob Chemother. 2012;67(6):1311-1320. doi:10.1093/jac/ dks065

4. Tacconelli E, Carrara E, Savoldi A, et al. Discovery, research, and development of new antibiotics: the WHO priority list of antibiotic-resistant bacteria and tuberculosis. Lancet Infect Dis. 2018;18(3):318-327. doi:10.1016/S1473-3099(17)30753-3

5. Chereau F, Opatowski L, Tourdjman M, Vong S. Risk assessment for antibiotic resistance in South East Asia. BMJ. 2017;358:j3393. doi:10.1136/ bmj.j3393

6. Myat TO, Hannaway RF, Zin KN, et al. ESBL- and carbapenemase-producing Enterobacteriaceae in patients with bacteremia, Yangon, Myanmar, 2014. Emerg Infect Dis. 2017;23(5):857-859. doi:10.3201/eid2305.161100

7. Aung MS, San N, Maw WW, et al. Prevalence of extended-spectrum beta-lactamase and carbapenemase genes in clinical isolates of Escherichia coli in Myanmar: dominance of bla(NDM-5) and emergence of bla(OXA-181). Microb Drug Resist. 2018;24(9):1333-1344. doi:10.1089/ mdr.2017.0387

8. Crandon JL, Ariano RE, Zelenitsky SA, Nicasio AM, Kuti JL, Nicolau DP. Optimization of meropenem dosage in the critically ill population based on renal function. Intensive Care Med. 2011;37(4):632-638. doi:10.1007/s00134-010-2105-0

9. Vinks A, Derendorf H, Mouton J, eds. Fundamentals of Antimicrobial Pharmacokinetics and Pharmacodynamics. New York: Springer; 2014.

10. Roberts JA, Kirkpatrick CM, Lipman J. Monte Carlo simulations: maximizing antibiotic pharmacokinetic data to optimize clinical practice for critically ill patients. J Antimicrob Chemother. 2011;66(2):227-231. doi:10.1093/jac/dkq449

11. Asín-Prieto E, Rodríguez-Gascón A, Isla A. Applications of the pharmacokinetic/pharmacodynamic (PK/PD) analysis of antimicrobial agents. $J$ Infect Chemother. 2015;21(5):319-329. doi:10.1016/j.jiac.2015.02.001

12. CLSI. Performance Standards for Antimicrobial Susceptibility Testing. 30th ed. CLSI Supplement M100. Wayne, PA: Clinical and Laboratory Standards Institute; 2020.

13. Thuy NTT, Thanh DD, Ha NH, Huong NTL. Population pharmacokinetics of meropenem in Vietnamese adult patients. Pharm Sci Asia. 2018;45 (4):221-230. doi:10.29090/psa.2018.04.017.0042

14. Li C, Du X, Kuti JL, Nicolau DP. Clinical pharmacodynamics of meropenem in patients with lower respiratory tract infections. Antimicrob Agents Chemother. 2007;51(5):1725-1730. doi:10.1128/AAC.00294-06

15. Pifizer (homepage on Internet). Merrem IV: US physician prescribing information; 2019. Avaliable from: https://www.pfizer.com/products/productdetail/merrem_i_v. Accessed January 18, 2022. 
16. European Medicines Agency (homepage on Internet). Meronem: article 30 referral - annex I, II, III; 2009. Avaliable from: https://www.ema.europa. eu/en/medicines/human/referrals/meronem\#overview-section. Accessed January 18, 2022.

17. Ehmann L, Zoller M, Minichmayr IK, et al. Development of a dosing algorithm for meropenem in critically ill patients based on a population pharmacokinetic/pharmacodynamic analysis. Int J Antimicrob Agents. 2019;54(3):309-317. doi:10.1016/j.ijantimicag.2019.06.016

18. Mendes RE, Mendoza M, Banga Singh KK, et al. Regional resistance surveillance program results for 12 Asia-Pacific nations (2011). Antimicrob Agents Chemother. 2013;57(11):5721-5726. doi:10.1128/AAC.01121-13

19. Suwantarat N, Carroll KC. Epidemiology and molecular characterization of multidrug-resistant gram-negative bacteria in Southeast Asia. Antimicrob Resist Infect Control. 2016;5:15. doi:10.1186/s13756-016-0115-6

20. Isla A, Canut A, Arribas J, Asín-Prieto E, Rodríguez-Gascón A. Meropenem dosing requirements against Enterobacteriaceae in critically ill patients: influence of renal function, geographical area and presence of extended-spectrum $\beta$-lactamases. Eur J Clin Microbiol Infect Dis. 2016;35 (3):511-519. doi:10.1007/s10096-015-2568-6

21. Mathew SK, Mathew BS, Neely MN, et al. A nonparametric pharmacokinetic approach to determine the optimal dosing regimen for 30-minute and 3-hour meropenem infusions in critically ill patients. Ther Drug Monit. 2016;38(5):593-599. doi:10.1097/FTD.0000000000000323

22. Guilhaumou R, Benaboud S, Bennis Y, et al. Optimization of the treatment with beta-lactam antibiotics in critically ill patients-guidelines from the French society of pharmacology and therapeutics (Société Française de Pharmacologie et Thérapeutique-SFPT) and the French society of anaesthesia and intensive care medicine (Société Française d'Anesthésie et Réanimation-SFAR). Crit Care. 2019;23(1):104. doi:10.1186/s13054019-2378-9

23. Gamper G, Havel C, Arrich J, et al. Vasopressors for hypotensive shock. Cochrane Database Syst Rev. 2016;2(2):Cd003709. doi:10.1002/ 14651858.CD003709.pub4

24. Bellomo R, Wan L, May C. Vasoactive drugs and acute kidney injury. Crit Care Med. 2008;36(4 Suppl):S179-S186. doi:10.1097/ CCM.0b013e318169167f

25. Ma S, Evans RG, Iguchi N, et al. Sepsis-induced acute kidney injury: a disease of the microcirculation. Microcirculation. 2019;26(2):e12483. doi: $10.1111 /$ micc. 12483

26. Lankadeva YR, Kosaka J, Evans RG, Bailey SR, Bellomo R, May CN. Intrarenal and urinary oxygenation during norepinephrine resuscitation in ovine septic acute kidney injury. Kidney Int. 2016;90(1):100-108. doi:10.1016/j.kint.2016.02.017

27. Asfar P, Teboul JL, Radermacher P, et al. High versus low blood-pressure target in septic shock. N Engl J Med. 2014;370(17):1583-1593. doi:10.1056/NEJMoa1312173

28. Grijalba AII, Aldaz Pastor A, Marquet P, Woillard JB. Evaluation of a non-parametric modelling for meropenem in critically ill patients using Monte Carlo simulation. Eur J Clin Pharmacol. 2019;75(10):1405-1414. doi:10.1007/s00228-019-02716-y

Infection and Drug Resistance

Dovepress

\section{Publish your work in this journal}

Infection and Drug Resistance is an international, peer-reviewed open-access journal that focuses on the optimal treatment of infection (bacterial, fungal and viral) and the development and institution of preventive strategies to minimize the development and spread of resistance. The journal is specifically concerned with the epidemiology of antibiotic resistance and the mechanisms of resistance development and diffusion in both hospitals and the community. The manuscript management system is completely online and includes a very quick and fair peer-review system, which is all easy to use. Visit http://www.dovepress.com/testimonials.php to read real quotes from published authors.

Submit your manuscript here: https://www.dovepress.com/infection-and-drug-resistance-journal 